Fluctuation characteristics of arc voltage and jet flow in a non-transferred dc plasma generated at reduced pressure

This article has been downloaded from IOPscience. Please scroll down to see the full text article.

2009 Plasma Sources Sci. Technol. 18045032

(http://iopscience.iop.org/0963-0252/18/4/045032)

The Table of Contents and more related content is available

Download details:

IP Address: 159.226.231.157

The article was downloaded on 22/12/2009 at 09:00

Please note that terms and conditions apply. 


\title{
Fluctuation characteristics of arc voltage and jet flow in a non-transferred dc plasma generated at reduced pressure
}

\author{
W X Pan ${ }^{1}$, Z Y Guo, X Meng, H J Huang and C K Wu \\ Institute of Mechanics, Chinese Academy of Sciences, Beijing 100190, People's Republic of China \\ E-mail: wxpan@imech.ac.cn
}

Received 3 May 2009, in final form 1 September 2009

Published 6 October 2009

Online at stacks.iop.org/PSST/18/045032

\begin{abstract}
A torch with a set of inter-electrode inserts between the cathode and the anode/nozzle with a wide nozzle exit was designed to generate plasma jets at chamber pressures of 500-10000 Pa. The variation of the arc voltage was examined with the change in working parameters such as gas flow rate and chamber pressure. The fluctuation in the arc voltage was recorded with an oscilloscope, and the plasma jet fluctuation near the torch exit was observed with a high-speed video camera and detected with a double-electrostatic probe. Results show that the $300 \mathrm{~Hz}$ wave originated from the tri-phase rectified power supply was always detected under all generating conditions. Helmholtz oscillations over $3000 \mathrm{~Hz}$ was detected superposed on the $300 \mathrm{~Hz}$ wave at gas flow rates higher than $8.8 \mathrm{slm}$ with a peak to valley amplitude lower than $5 \%$ of the average voltage value. No appreciable voltage fluctuation caused by the irregular arc root movement is detected, and mechanisms for the arc voltage and jet flow fluctuations are discussed.
\end{abstract}

(Some figures in this article are in colour only in the electronic version)

\section{Introduction}

Non-transferred direct current (dc) plasmas are widely used as a heating source for various purposes such as materials processing, high temperature wind tunnel and special waste treatment. Fluctuation characteristics of the jet flow seem to affect the uniform heating of precursors or particles injected into it continuously in a film deposition or spray coating process $[1,2]$, causing an increase in the structural defects in the film or coating and making it difficult to control their properties. Reports show that the fluctuation characteristics in the jet flow near the torch exit are generally similar to that of an arc voltage [3-5], according to the simultaneous detection of light emission intensity from the jet flow and the measurement of arc voltage. That is, the change in the arc voltage modifies the input energy to plasma generation, which causes different gas heating effects and brings about the fluctuation of the parameters in the jet flow.

Generally, the possible mechanisms causing the arc voltage fluctuation can be mainly divided into three different

\footnotetext{
1 Author to whom any correspondence should be addressed.
}

types [4-8]. The most common type could be that caused by the length change of the arc column between the cathode tip and the anode, due to the arc root movement on the anode surface owing to the interaction between the drag force of gas flow and the electro-magnetic force around the arc column. Many factors could affect the attachment pattern and the form of movement of the arc root on the anode surface, such as the working gas component, feeding method, flow rate, arc current and boundary layer between the anode surface and the arc column $[6,7,9]$. The amplitude and frequency of the fluctuation change in a wide range and can be over $100 \%$ of its average voltage and several kilohertz $[7,8,10]$. Another type of arc voltage fluctuation is considered originating from the power supply. The fluctuation amplitude of the arc voltage is generally lower than $15 \%$ of its average value with a characteristic frequency around $100-300 \mathrm{~Hz}[4,5,11]$, which reflects the output characteristics of the rectified power supply. For a chosen power supply, the fluctuation characteristic frequency is fixed at a certain value, and the voltage always fluctuated at the frequency with a limited amplitude change with the change in gas flow rate or arc current. This kind 
of fluctuation can be removed only by changing the power supply with minimized ripple characteristics. The third type of fluctuation seems to arise from Helmholtz oscillations affected mainly by the torch geometry and also the gas flow condition and input power level $[4,8]$. In this case, the torch gas flow channel appears as a Helmholtz resonator, which allows a resonance to occur at a certain frequency determined by the channel structure and operating conditions. This kind of resonance is generally superposed on the other two types of fluctuations with its own characteristic frequency and amplitude.

Regarding the first type of arc voltage fluctuation, a fixed arc length may be preferred to ensure stable arcing which can be achieved by an abruptly expanded channel of the torch design [12]. Besides, between the cathode and the anode of the non-transferred dc plasma torch, an insert of floating electric potential with a relative long, narrow tunnel through which the arc column passes was added to generate the plasma under an almost fixed arc length condition [13-15]. That is, with the special torch structure, the arcing can only find its suitable attachment position on the anode surface within an almost fixed and narrow region at a given gas feeding condition and arc current. In this kind of torch, the change in arc column length caused by the movement of the arc root on the anode surface could generally be neglected compared with its long column passing through the inter-electrode insert. Thus, the fluctuation component of the arc voltage originating from the length change of arc column can be kept generally at a negligible level [4]. Experimental results indicate that this kind of torch can generate a plasma jet of laminar or turbulent flow state at atmospheric pressure by regulating the gas flow rate and arc current [14]. When a laminar plasma jet was generated, only the voltage fluctuation originated from the feature of the power supply was observed [4]. This kind of laminar plasma jet can be used for the surface treatment of metals, such as re-melting hardening, cladding and spray coating $[16,17]$, with greatly improved process controllability compared with that by using the turbulent plasma jet.

In this work, a torch with a set of inter-electrode inserts between the cathode and the anode of a wide nozzle exit was designed to generate dc thermal plasma of larger volume and moderate parameter gradient at reduced pressures. The voltage characteristics of the torch and the fluctuation in arc voltage and plasma jet flow were examined with the change in working parameters such as gas flow rate and chamber pressure.

\section{Plasma generation}

Plasma jets were generated with the torch shown schematically in figure 1 by using pure argon as the working gas at a fixed arc current of $80 \mathrm{~A}$. A tri-phase rectified dc power supply capable of $20 \mathrm{~kW}$ output was used. Gas is injected tangentially near the cathode tip and also at the entrance to the anode cavity. The inter-electrode insert in figure 1 comprises three copper inserts and two insulator inserts in between. The inserts have a floating electric potential and an inside diameter of $8 \mathrm{~mm}$ to form an arc channel through which the arc column just passes but cannot find its attachment spot on the channel surface. The

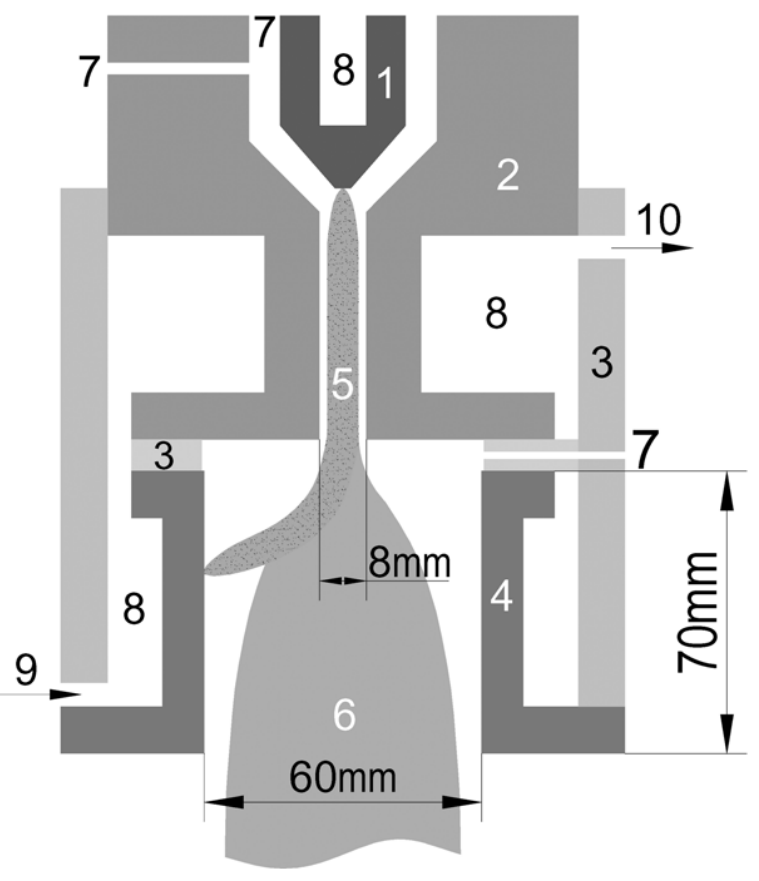

Figure 1. Schematic diagram of the plasma torch. 1, cathode; 2, inter-electrode insert; 3 , insulator; 4 , anode; 5 , arc column; 6 , plasma jet; 7 , gas inlet; 8 , cooling water; 9 , water inlet; 10 , water outlet.

anode nozzle has an inside diameter of $60 \mathrm{~mm}$ which is much wider than the inter-electrode channel. It is predicted that the drag force of the flow in the wide nozzle region is too weak for driving the arc root movement intensively and producing arc voltage fluctuation of the restrike mode, under the present working conditions of gas flow rate and chamber pressure.

The appearances of the plasma jet flow taken by an ordinary camera are shown in figure 2 . The shutter time for each photo is $1 / 200 \mathrm{~s}$. The luminous region of the jet flow expanded with decreasing chamber pressure and the light intensity at the centre of torch exit increased with increasing chamber pressure. These suggest that the plasma volume increased and the temperature gradient decreased with decreasing chamber pressure. Such kinds of plasmas operated at a reduced pressure may have advantages for certain kinds of materials processing where large area and uniform treatment are preferred.

The arc voltage increases with increasing gas flow rate or, more gently, with increasing chamber pressure, as indicated in figure 3. This dependence of the arc voltage change on the gas flow rate was also observed when a similar torch with only one piece of inter-electrode insert and relatively narrower nozzle diameter was operated at atmospheric pressure [4]. The arc voltage is generally related to the column length and the intensity of the electric field $E=j / \sigma$. Here $j$ is the arc current density and $\sigma$ is the electric conductivity, and usually arc constriction leads to a higher $E$. In the case of the present torch, the length change of the arc column can almost be neglected. Accordingly, the characteristic of the arc voltage in figure 3 indicates that $E$ varies with chamber pressure and gas flow rate. Previous pressure measurements show that when the gas flow rate increases from 6.8 to $17.6 \mathrm{slm}$, the pressure in the upstream cathode cavity increases from 12000 to $27000 \mathrm{~Pa}$. 


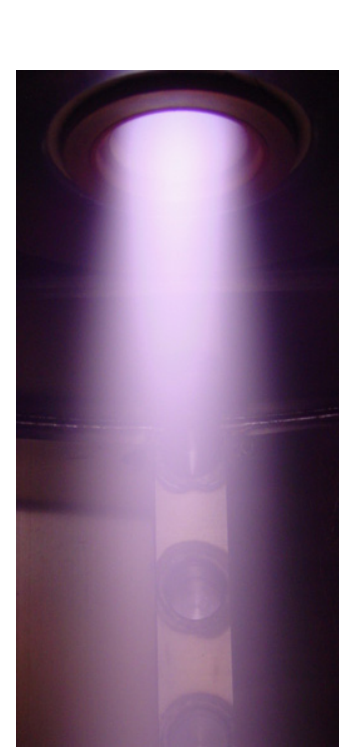

(a)

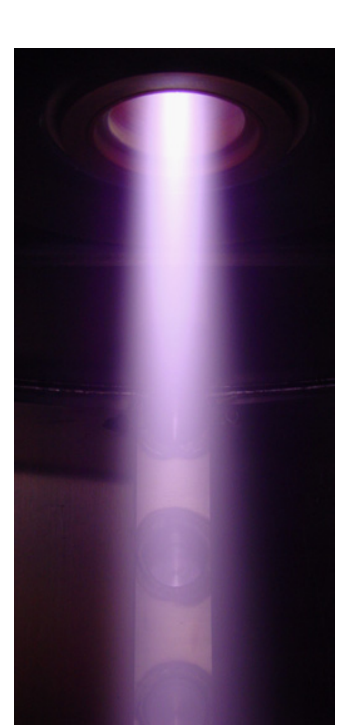

(b)

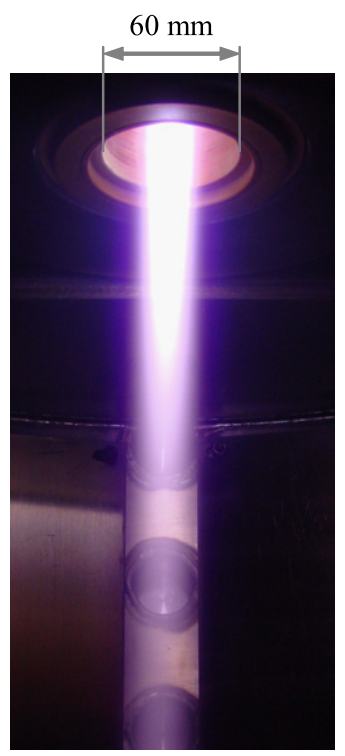

(c)

Figure 2. Photographs showing the appearance of the pure argon plasma jets at a gas flow rate of $4.4 \mathrm{~s} / \mathrm{m}$ and chamber pressures of (a) $170 \mathrm{~Pa},(b) 500 \mathrm{~Pa}$ and (c) $2000 \mathrm{~Pa}$. The shutter time of the camera is $1 / 200 \mathrm{~s}$.

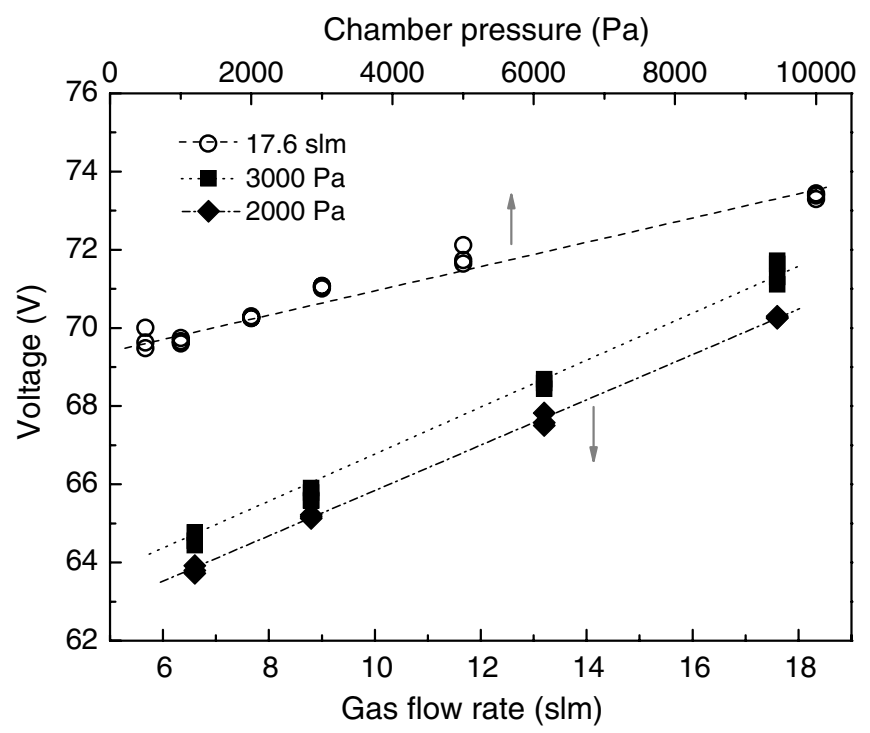

Figure 3. Variations of the mean arc voltage with gas flow rate at fixed chamber pressures of $2000 \mathrm{~Pa}$ and $3000 \mathrm{~Pa}$ and with chamber pressure at a fixed gas flow rate of $17.6 \mathrm{slm}$.

However, when the gas flow rate is constant, it is independent of the plenum chamber pressure in this study; e.g. at a gas flow rate of $17.6 \mathrm{slm}$, the pressure in the cathode cavity remains constant at $27000 \mathrm{~Pa}$ when the plenum chamber pressure is decreased to values below $10000 \mathrm{~Pa}$. This is the phenomenon known as thermal choking [19]. The velocity of the jet has reached the speed of sound at the exit of the inter-electrode insert, and the downstream pressure does not affect the flow inside the insert channel. Therefore, the measured arc voltage can be divided into two parts: that of the arc inside the interelectrode channel which varies only with gas flow rate and that of the portion in the anode cavity which changes with both gas flow rate and chamber pressure. Generally, higher gas flow rates lead to more arc column constriction, thus to elevated arc voltage. With the variation of the plenum chamber pressure, although the first part of the arc does not change, the part of the arc column in the anode cavity does become more constricted at higher pressures, also leading to an increase in the arc voltage.

\section{Experimental measurement and data analysing methods}

The time-resolved arc voltage signals between the torch anode and cathode in generating plasma jets were obtained directly by using an oscilloscope with a sampling rate of $500 \mathrm{kHz}$. A highspeed video camera capable of $1.2 \times 10^{5}$ frame per second (fps) was used to obtain the instantaneous plasma jet images through the chamber window, with a recording speed of $2500 \mathrm{fps}$ and an exposure time of $10 \mu$ s per frame, while the lens aperture was set to 0.95 .

A double-electrostatic probe system was set at the jet centre near the torch exit to measure the ion saturation current. The probe system consists mainly of five parts as shown in figure 4. That is, a double-electrostatic probe, one probe holder, a dc bias power supply, a sampling resistance of $500 \Omega$ and an oscilloscope. The double-electrostatic probe comprises two individual tungsten wire electrodes of $0.3 \mathrm{~mm}$ diameter with an exposed length of $2 \mathrm{~mm}$ in the plasma. The other part of each wire is covered by an alumina tube of $0.5 \mathrm{~mm}$ inner diameter, $1 \mathrm{~mm}$ outer diameter and $35 \mathrm{~mm}$ length. The probe head is fixed in a boron nitride holder with a centre distance of $2 \mathrm{~mm}$ between the two wires. The holder can move along the axial and radial directions of the jet. The bias power supply applies a voltage of $18 \mathrm{~V}$ between these two tungsten wires, and the ion current is calculated by the voltage collected across the resistance. It was confirmed that the ion current reached its saturation value at the bias voltage of $18 \mathrm{~V}$ under the working conditions. An oscilloscope with an isolated channel was used to record the time-resolved voltage signals across the resistance with a sampling rate of $100 \mathrm{kHz}$. 


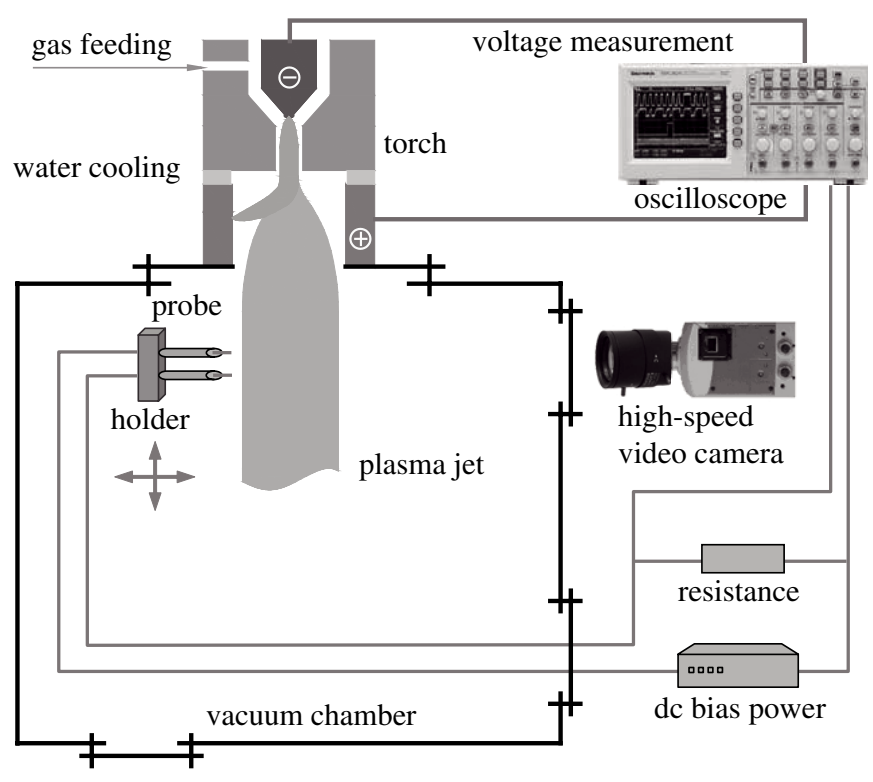

filtering \& vacuum system

Figure 4. Schematic diagram of the experimental setup.

Fast Fourier transform (FFT) was applied to analyse the detected arc voltage spectra and ion saturation current spectra for discussing the characteristic frequency of the fluctuation in the arc column and jet flow.

\section{Results and discussions}

The measurement results of fluctuation conditions for arc voltage and plasma jet flow depending on the generating parameters such as gas flow rate and chamber pressure are illustrated and analysed. Correlations between jet flow state and the situation of arc voltage fluctuation are discussed.

\subsection{Effects of gas flow rate and chamber pressure on the arc voltage fluctuation}

The arc voltage shows low frequency waves in the whole range of gas flow rate as in figure 5 when the chamber pressure is fixed at $3000 \mathrm{~Pa}$. At a low gas flow rate of $4.4 \mathrm{slm}$, there exists only a low frequency wave in the arc voltage spectra. As the gas flow rate increases, a high-frequency wave appears, superposed on the low frequency one with a smaller amplitude and becomes increasingly clear. FFT results at gas flow rates of 4.4, 8.8 and $17.6 \mathrm{slm}$ corresponding to the data in figure 5 are shown in figure 6 . It indicates that the low frequency wave is at $300 \mathrm{~Hz}$, which reflects the characteristics of the power supply and appears in all cases regardless of the generating condition [4]. It will be called 'power fluctuation' in the following discussions. There is a clear peak at frequency near $3160 \mathrm{~Hz}$ for the arc voltage generated at a gas flow rate of $17.6 \mathrm{slm}$, but no high-frequency component could be found at a gas flow rate of $4.4 \mathrm{slm}$. A weak peak appeared at about $3460 \mathrm{~Hz}$ at the flow rate of $8.8 \mathrm{slm}$, indicating the existence of a high-frequency fluctuation under this working condition.

The effect of chamber pressure is different from that of the gas flow rate. Figure 7 shows that the high-frequency wave

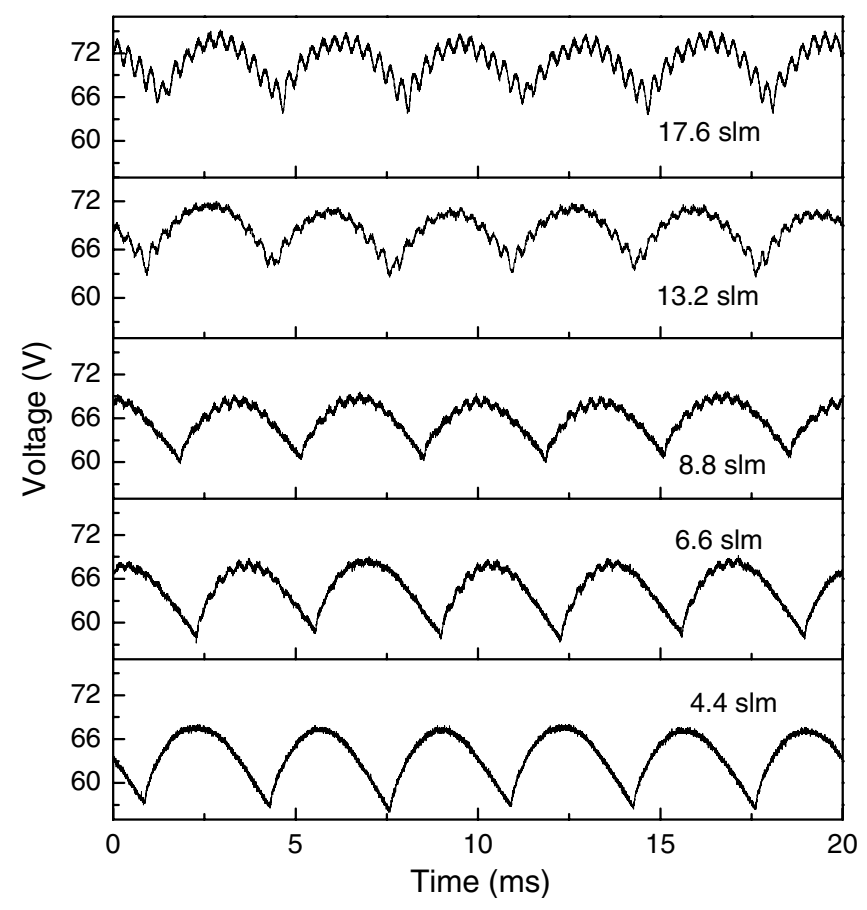

Figure 5. Time-resolved arc voltages at a chamber pressure of $3000 \mathrm{~Pa}$ and different gas flow rates of $17.6 \mathrm{slm}, 13.2 \mathrm{slm}, 8.8 \mathrm{slm}$, $6.6 \mathrm{slm}$ and $4.4 \mathrm{slm}$.

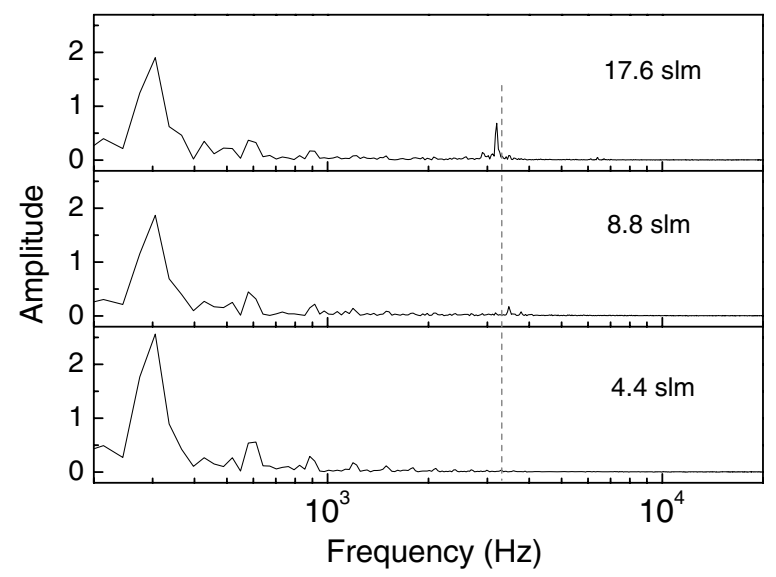

Figure 6. FFT results for arc voltage spectra at a chamber pressure of $3000 \mathrm{~Pa}$ and gas flow rates of $17.6 \mathrm{slm}, 8.8 \mathrm{slm}$ and $4.4 \mathrm{slm}$.

exists clearly at all chamber pressures ranging from 500 to $10000 \mathrm{~Pa}$, when the gas flow rate is fixed at the high level of $17.6 \mathrm{slm}$. Figure 8 is the FFT results corresponding to the voltage data in figure 7 at chamber pressures of 500 and $10000 \mathrm{~Pa}$. A sharp and clear peak appears at a frequency of about $3160 \mathrm{~Hz}$ under the two working conditions at the very different chamber pressures. That is, unlike the effect of gas flow rate on the high-frequency fluctuation of arc voltage, chamber pressure shows almost no effects on the existence and frequency of the high-frequency fluctuation component in the arc voltage.

Figure 9 summarizes the FFT analysis results for the highfrequency fluctuation in the arc voltage. The horizontal dashed line in the figure is an arithmetic mean of the frequency values, which remains at about $3180 \mathrm{~Hz}$ in the whole chamber pressure 


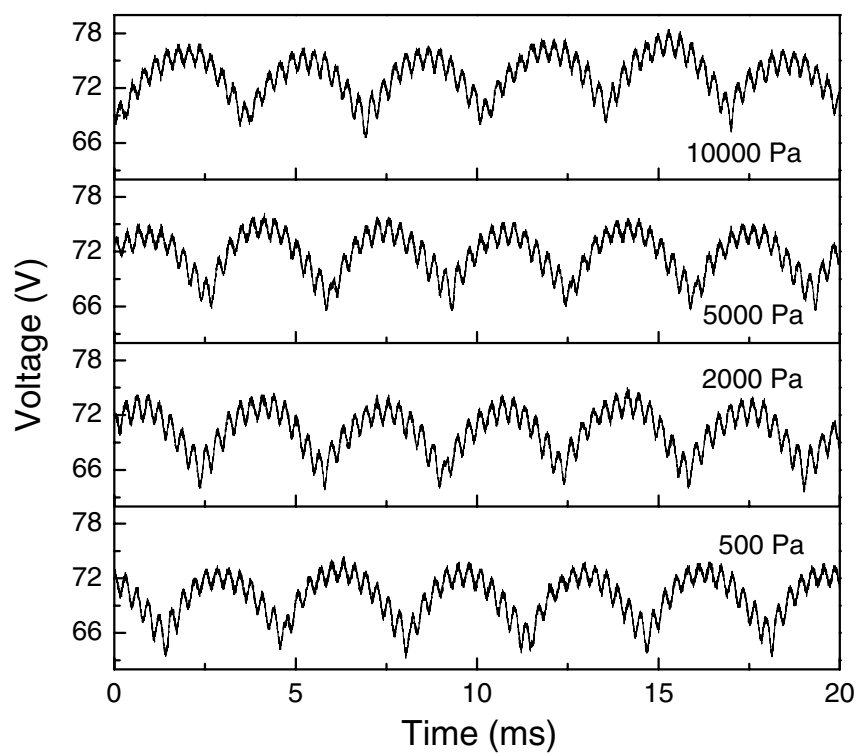

Figure 7. Time-resolved arc voltages at a gas flow rate of $17.6 \mathrm{slm}$ and different chamber pressures of $10000 \mathrm{~Pa}, 5000 \mathrm{~Pa}, 2000 \mathrm{~Pa}$ and $500 \mathrm{~Pa}$.

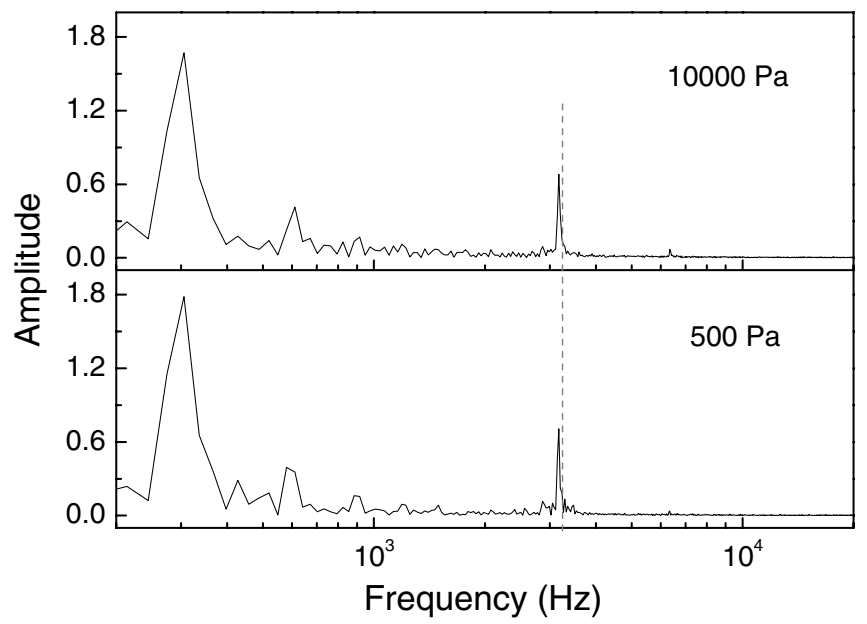

Figure 8. FFT results for arc voltage spectra at a gas flow rate of $17.6 \mathrm{slm}$ and different chamber pressures of $10000 \mathrm{~Pa}$ and $500 \mathrm{~Pa}$.

range from 500 to $10000 \mathrm{~Pa}$ when the gas flow rate is fixed at $17.6 \mathrm{slm}$. The solid line represents the experimental results at chamber pressures of 2000 and $3000 \mathrm{~Pa}$ as the gas flow rate changes from 4.4 to $17.6 \mathrm{slm}$. The fluctuation frequency decreases with increasing gas flow rate monotonically. This suggests that chamber pressure has no effect on the fluctuation frequency in the entire gas flow rate range in this work.

Setting the maximum value of the peak to valley of the high-frequency fluctuation as $\Delta V_{\max }$, and the mean value of the arc voltage as $V$, figures 10 and 11 show that variations of $\Delta V_{\max }$ and $\Delta V_{\max } / V$ change with the gas flow rate and chamber pressure. In figure $10, \Delta V_{\max }$ and $\Delta V_{\max } / V$ both increased with increasing gas flow rate when the chamber pressure was fixed at $2000 \mathrm{~Pa}$, and the highest values are about $3.3 \mathrm{~V}$ and $4.6 \%$, respectively, at the high gas flow rate of $17.6 \mathrm{slm}$. However, $\Delta V_{\max }$ and $\Delta V_{\max } / V$ both decreased slightly with increasing chamber pressure when the gas flow rate was fixed at $17.6 \mathrm{slm}$ as shown in figure 11 . This could be

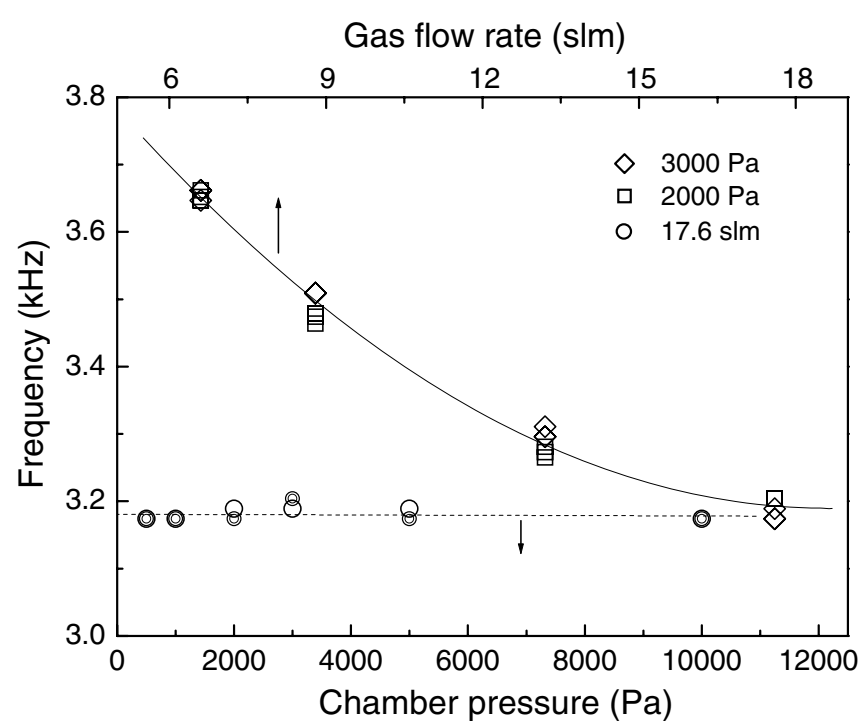

Figure 9. Variations of the characteristic frequency of high-frequency fluctuation in arc voltage with the change in chamber pressure at a fixed gas flow rate of $17.6 \mathrm{slm}$ and with gas flow rate at chamber pressures of $2000 \mathrm{~Pa}$ and $3000 \mathrm{~Pa}$.

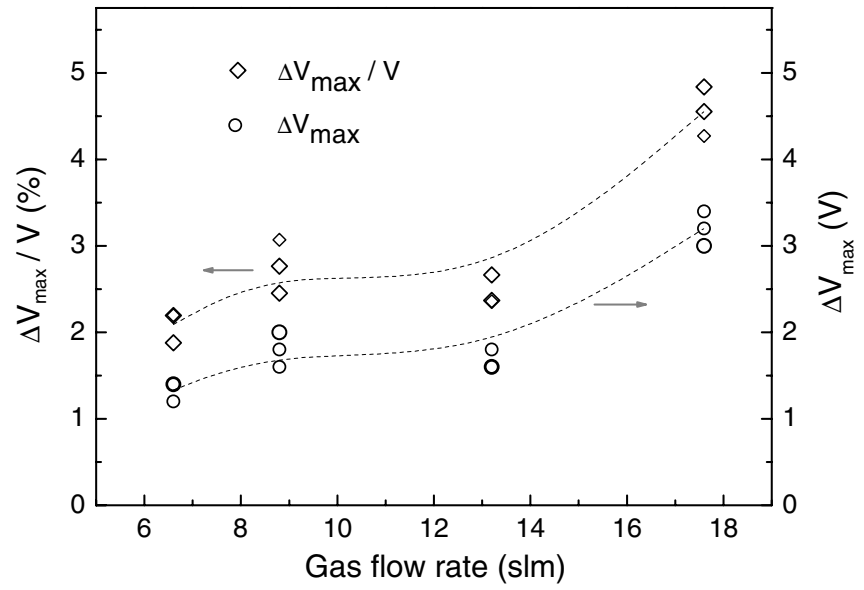

Figure 10. Variations of the maximum amplitude of high-frequency fluctuation in the arc voltage and the ratio of the maximum fluctuation to mean arc voltage with the gas flow rate at a chamber pressure of $2000 \mathrm{~Pa}$.

due to the decrease in the actual flow velocity in the channel with the increase in chamber pressure when the feeding flow rate is kept unchanged.

\subsection{Effects of gas flow rate and chamber pressure on the condition of the jet flow}

The images of the visible plasma jets outside the torch nozzle taken by the high-speed video camera are shown in figure 12 . In the case of low gas flow rates of 4.4 and $6.6 \mathrm{slm}$ at the chamber pressure of $2000 \mathrm{~Pa}$, no appreciable swinging can be seen in the luminous region of the jet flow even when the exposure time is as short as $10 \mu \mathrm{s}$, which indicates that the plasma jet could be in a stable laminar flow state. As the gas flow rate increases, the high temperature region represented by the high intensity light emission area in the image swung around irregularly. This is in step with the effect of gas flow rate on the fluctuation of 


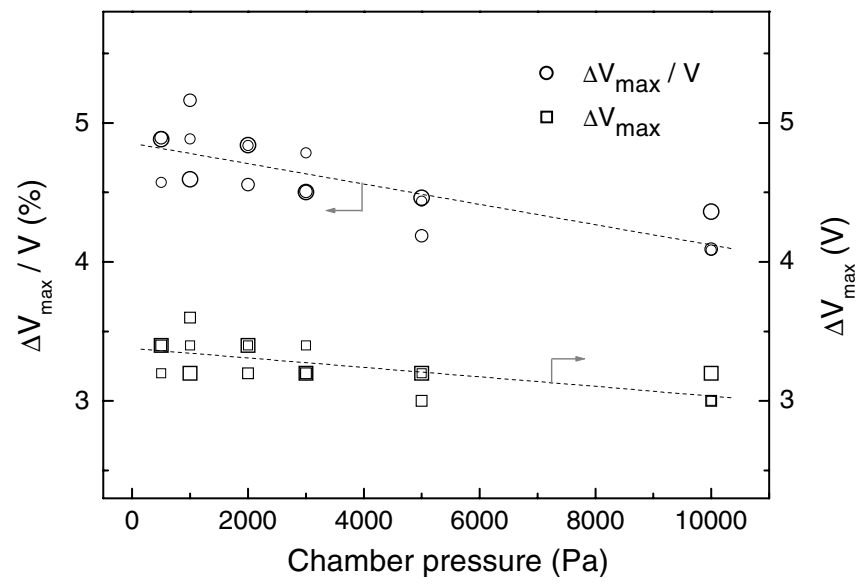

Figure 11. Variations of the maximum amplitude of high-frequency fluctuation in the arc voltage and the ratio of the maximum fluctuation to mean arc voltage with the chamber pressure at a gas flow rate of $17.6 \mathrm{slm}$.
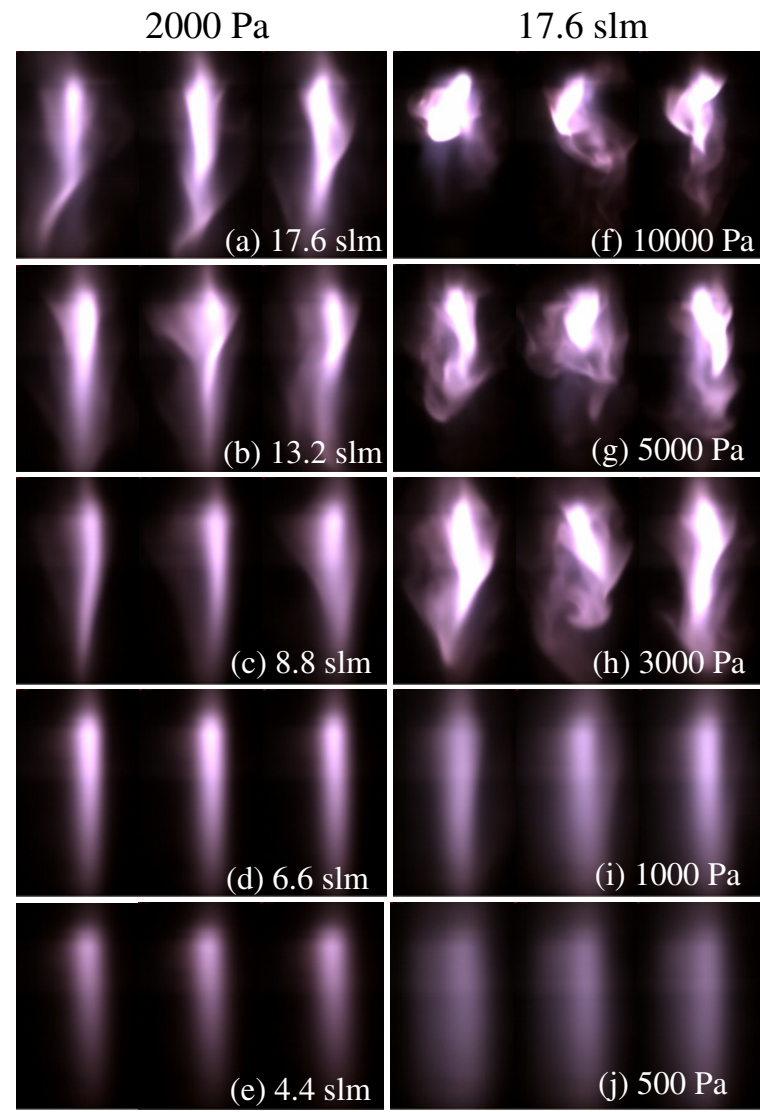

Figure 12. High-speed video camera images of the plasma jet generated under different working conditions. $(a)-(e)$ at chamber pressure of $2000 \mathrm{~Pa},(a)$ at gas flow rate of $17.6 \mathrm{slm},(b) 13.2 \mathrm{slm}$, $(c) 8.8 \mathrm{slm},(d) 6.6 \mathrm{slm}$ and $(e) 4.4 \mathrm{slm} ;(f)-(j)$ at gas flow rate of $17.6 \mathrm{slm},(f)$ at chamber pressure of $10000 \mathrm{~Pa},(g) 5000 \mathrm{~Pa}$, (h) $3000 \mathrm{~Pa}$, $(i) 1000 \mathrm{~Pa}$ and $(j) 500 \mathrm{~Pa}$. The recording rate of the camera was $2500 \mathrm{fps}$ and the exposure time was $10 \mu \mathrm{s}$ for each image, three images under the same working condition are taken in successive sequence.

arc voltage shown in figure 5, in which the high-frequency fluctuation becomes evident as the gas flow rate increased above $6.6 \mathrm{slm}$. However, luminous images of the jet flow at 500 and $1000 \mathrm{~Pa}$ in figures $12(i)$ and $(j)$ also show good stability

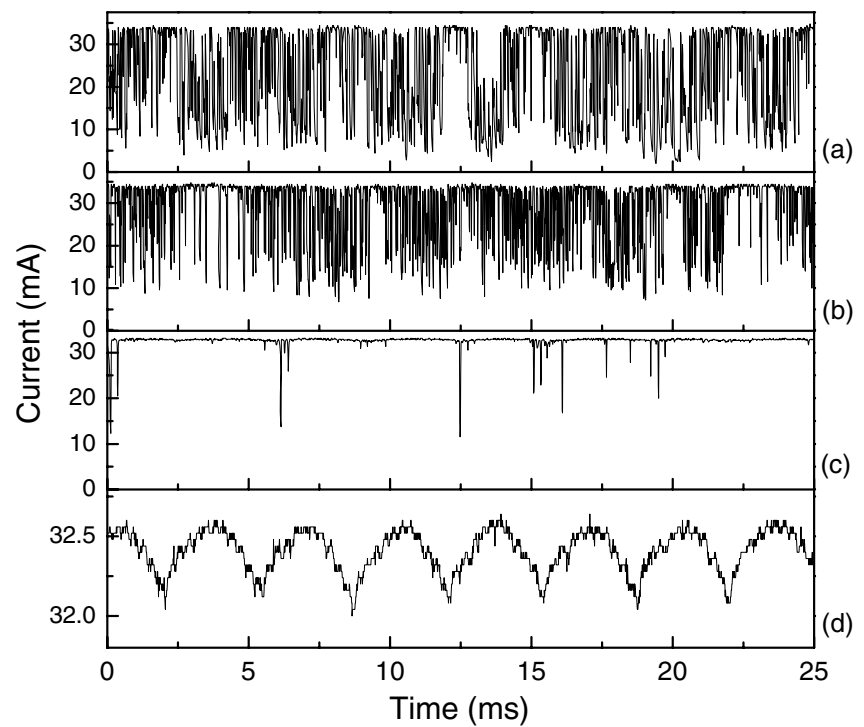

Figure 13. Time-resolved ion saturation current captured by the electrostatic probe at a gas flow rate of $17.6 \mathrm{slm}$ and chamber pressures of (a) $10000 \mathrm{~Pa},(b) 5000 \mathrm{~Pa},(c) 2000 \mathrm{~Pa}$ and (d) $500 \mathrm{~Pa}$.

and uniformity at the high gas flow rate of $17.6 \mathrm{slm}$, while there is a clear high-frequency fluctuation in their arc voltage as in figure 7 and the fluctuation amplitude $\left(\Delta V_{\max }\right)$ is slightly higher than that generated at higher chamber pressures as in figure 11. This suggests that arc voltage fluctuations do not necessarily result in jet flow instability at these low pressures, and the two phenomena are not always directly related as cause and effect.

Figure 13 shows the time-resolved ion saturation current passing through the double-probe circuit set in the jet flow generated at different chamber pressures of 500, 2000, 5000 and $10000 \mathrm{~Pa}$ at the fixed gas flow rate of $17.6 \mathrm{slm}$. The signal fluctuation increased with increasing chamber pressure. At the chamber pressure of $500 \mathrm{~Pa}$, it shows a completely regular wave with about $1.8 \%$ amplitude fluctuation, which is similar to that of the arc voltage in figure 7 . When the chamber pressure was at $2000 \mathrm{~Pa}$, sudden jumping of the signal occurred occasionally. As the chamber pressure increased to $5000 \mathrm{~Pa}$, the ion saturation current fluctuated violently almost all the time, and sometimes showed a very low value near zero at the chamber pressure of $10000 \mathrm{~Pa}$. This suggests that the high temperature region with relative high ionization and electron density swung at that instant away from the location at which the probe was set.

Figure $14(a)$ is the FFT analysis results for the ion saturation current shown in figure 13 at low chamber pressures of 500 and $2000 \mathrm{~Pa}$. Similar to the situation for the arc voltage in figure 8 , the peaks near $300 \mathrm{~Hz}$ owing to the power fluctuation and near $3160 \mathrm{~Hz}$ for the high-frequency fluctuation exist clearly at the chamber pressure of $500 \mathrm{~Pa}$. This indicates that the plasma jet flow completely inherited and maintained the fluctuation characteristics of the arc voltage, and no other surge and turbulence factors were introduced to the plasma jet flow under this condition. As the chamber pressure increased to $2000 \mathrm{~Pa}$, noises appear in almost whole frequency range but no evident peak is distinguished at the high-frequency side. 

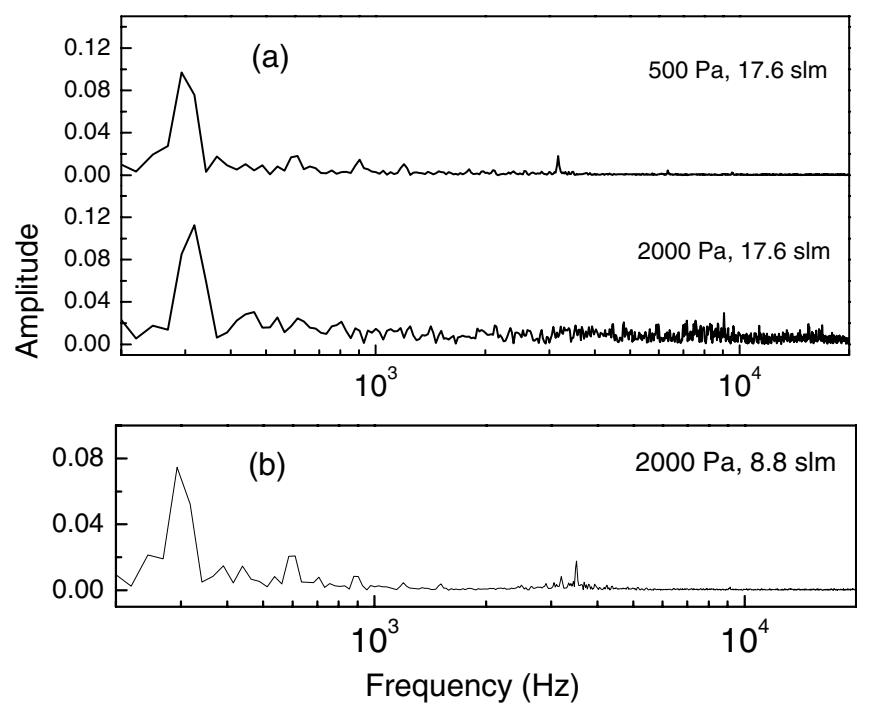

Figure 14. FFT results for ion saturation current captured by the electrostatic probe at $(a)$ gas flow rate of $17.6 \mathrm{slm}$ (chamber pressures of $500 \mathrm{~Pa}$ and $2000 \mathrm{~Pa}$ ) and $(\mathrm{b})$ at chamber pressure of $2000 \mathrm{~Pa}$ and gas flow rate of $8.8 \mathrm{slm}$

When the chamber pressure increases to $5000 \mathrm{~Pa}$, the signal shows a completely irregular fluctuation of larger amplitude. At the low gas flow rate of $8.8 \mathrm{slm}$ and chamber pressure of $2000 \mathrm{~Pa}$, peaks at 300 and $3490 \mathrm{~Hz}$ can be seen as in figure $14(b)$.

\subsection{Discussion}

It has been mentioned that the arc voltage fluctuation generally arises from three causes. In this work, the $300 \mathrm{~Hz}$ fluctuation is caused by the power fluctuation. It was determined that the high-frequency fluctuation over $3000 \mathrm{~Hz}$ did not match the irregular arc root movement observed in the previous work using the same torch and power supply [18], but Helmholtz oscillation seems to be a reasonable cause. According to the analysis of Coudert et al [20], the square of the Helmholtz oscillation frequency is proportional to the specific enthalpy $h$ and to the gas pressure ratio of $P_{0} / P$. Here $P_{0}$ is the pressure in the cold cavity upstream of the arc column and $P$ is the pressure in the nozzle channel. Figure 9 indicates that the change in chamber pressure does not affect the oscillation frequency at a fixed gas flow rate, which means that $P_{0} / P$ and the arc voltage (hence, input power) were all changed insignificantly and resulted in a negligible effect on the frequency as the chamber pressure is changed. On the other hand, taking the situation at $2000 \mathrm{~Pa}$ as the example, figure 3 shows that the arc voltage increases from 63.9 to $70.2 \mathrm{~V}$, a change of $9.9 \%$, at a fixed arc current of $80 \mathrm{~A}$, as the gas flow rate increases from 4.4 to $17.6 \mathrm{slm}$, an increase of $300 \%$. The specific enthalpy of the flow would decrease quite appreciably and hence the oscillation frequency decreases with increasing gas flow rate clearly as shown in figure 9 .

The fluctuations of the light signal at the torch exit generally show similar characteristics as the arc voltage [3,5]. However, only the stable or laminar flows at the low chamber pressures and/or low gas flow rates show similar fluctuation characteristics between their arc voltage and electrostatic probe signal as shown in figures 7 and $13(d)$ of this work. With the increase in chamber pressure, plasma volume decreases and the high temperature region becomes shortened. And as the gas flow rate increases, the plasma jet flow transits from the stable laminar to a turbulent state. The potential core of the turbulent plasma jet becomes much shortened and locates completely within the long anode nozzle channel and is out of the visible region in the side view. Thus, as the chamber pressure and the gas flow rate increase, the electrostatic probe detects the ion saturation current in the downstream flow after the potential core of the turbulent plasma jet, in which the signal fluctuated irregularly in large scale as shown in figures $13(a)$ and $(b)$ at chamber pressures of 10000 and $5000 \mathrm{~Pa}$.

The results indicate that the laminar or turbulent state of the plasma jet flow bears no direct dependence on the power fluctuation and Helmholtz oscillation of its arc voltage. For example, although the laminar flow at $500 \mathrm{~Pa}$ and the thoroughly developed turbulent flow at $10000 \mathrm{~Pa}$ show similar fluctuation characteristics of their arc voltage as in figures 7 and 8, their jet appearance and electrostatic signal indicate quite different flow characteristics as in figures 12 and 13. Other factors besides arc voltage fluctuations, such as aerodynamic criteria, might also decidedly affect the state of the jet flow.

With the power supply used in the present setup, the fluctuation originated from the power supply always exists regardless of the torch structure and working parameters, but would be eliminated by selecting or improving the power units. The voltage amplitude of the Helmholtz oscillation is lower than $3.5 \mathrm{~V}$, within $5 \%$ of the average voltage value. Accordingly, it could be expected that this kind of oscillation would not become the dominant factor affecting the controllability in materials processing. However, the plasma jet flow state of laminar or turbulent condition could be an important factor affecting the process controllability. One can expect that the general parameter estimation by experimental measurement or numerical simulation for a turbulent jet flow could only give a rough and time-averaged outline according to the results shown in figures 12 and 13, and it is difficult to achieve precise process control under turbulent flow conditions.

\section{Conclusions}

The results of the study on this type of plasma generator show that the arc voltage increases with increasing gas flow rate and, more gently, with increasing chamber pressure. The Helmholtz oscillations above $3000 \mathrm{~Hz}$ are detected superposed on the $300 \mathrm{~Hz}$ power fluctuation at high gas flow rates, and the oscillation frequency decreases monotonically with increasing gas flow rate and remains unchanged with the chamber pressure. The voltage amplitude of the oscillation is lower than $3.5 \mathrm{~V}$, within $5 \%$ of the average voltage value, which should not have important effects on the controllability in materials processing. It is predicted that the long and narrow inter-electrode inserts mainly decided the length of the arc column, and the drag force of the flow in the wide nozzle 
region is too weak for driving the arc root movement to produce arc voltage fluctuations of the restrike mode. The laminar or turbulent state of jet flow shows no definite dependence on the power fluctuation of $300 \mathrm{~Hz}$ and Helmholtz oscillation in its arc voltage. The laminar jets at low gas feeding rates and/or low chamber pressures show steady flows while the turbulent jet flows at high gas flow rates and high chamber pressures swing around irregularly. Aerodynamic criteria downstream of the arc root attachment in the nozzle channel might decidedly affect the state of the jet flow. It is expected that the turbulent flow state could be a very important factor limiting the controllability for materials processing.

\section{Acknowledgment}

This work is supported by the National Natural Science Foundation of China (Nos 50836007, 10621202).

\section{References}

[1] Nogues E, Vardelle M, Fauchais P and Granger P 2008 Surf. Coat. Technol. 2024387

[2] Kuz'min V, Solonenko O and Zhukov M 1995 Proc. 8th National Thermal Spray Conference (Houston, TX) p 83

[3] Dorier J L, Hollenstein Ch, Salito A, Loch M and Barbezat G 1999 Proc. 14th Int. Symp. on Plasma Chemistry (Prague, Czech Republic) vol 1 p 331

[4] Pan W X, Meng X and Wu C K 2006 Plasma Sci. Technol. 8416
[5] Zhao W H, Tian K, Tang H Z, Liu D and Zhang G Z 2002 J. Phys. D: Appl. Phys. 352815

[6] Duan Z and Heberlein J 2002 J. Thermal Spray Technol. 1144

[7] Ghorui S, Vysohlid M, Heberlein J and Pfender E 2007 Phys. Rev. E 76016404

[8] Coudert J F and Rat V 2008 J. Phys. D: Appl. Phys. 41205208

[9] Pan W X, Meng X, Li T, Chen X and Wu C K 2007 Plasma Sci. Technol. 9152

[10] Brilhac J F, Pateyron B, Coudert J F, Fauchais P and Bouvier A 1995 Plasma Chem. Plasma Process. 15257

[11] Tu X, Cheron B G, Yan J H and Cen K F 2007 Plasma Sources Sci. Technol. 16803

[12] Zhukov M F and Zasypkin I M 2007 Thermal Plasma Torches-Design, Characteristics, Applications (Cambridge, UK: Cambridge International Science Publishing)

[13] Pan W X, Zhang W Hong, Zhang W Hua and Wu C K 2001 Plasma Chem. Plasma Process. 2123

[14] Pan W X, Meng X, Chen X and Wu C K 2006 Plasma Chem. Plasma Process. 26335

[15] Pan W X, Lu F X, Tang W Z, Zhong G F, Jiang Z and Wu C K 2000 Diamond Relat. Mater. 91682

[16] Pan W X, Meng X, Li G., Fei Q X and Wu C K 2005 Surf. Coat. Technol. 197345

[17] Pan W X, Ma W and Wu C K 2001 Proc. Int. Symp. of Young Scholars on Mechanics and Material Engineering for Science and Experiments (Changsha, China) p 427

[18] Huang H J, Pan W X and Wu C K 2008 Chin. Phys. Lett. 254058

[19] Pouring A A 1965 Phys. Fluids 81802

[20] Coudert J F, Rat V and Digot D 2007 J. Phys. D: Appl. Phys. 407357 